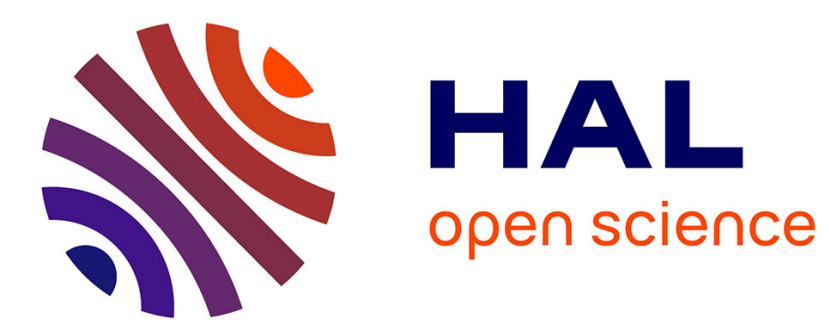

\title{
Integral sliding mode control of an extended Heisenberg system
}

Michael Defoort, Thierry Floquet, Wilfrid Perruquetti, Serguey Drakunov

\section{To cite this version:}

Michael Defoort, Thierry Floquet, Wilfrid Perruquetti, Serguey Drakunov. Integral sliding mode control of an extended Heisenberg system. IET Control Theory and Applications, 2009, 3 (10), pp.1409-1424. 10.1049/iet-cta.2007.0480 . inria-00366042

\section{HAL Id: inria-00366042 \\ https://hal.inria.fr/inria-00366042}

Submitted on 5 Mar 2009

HAL is a multi-disciplinary open access archive for the deposit and dissemination of scientific research documents, whether they are published or not. The documents may come from teaching and research institutions in France or abroad, or from public or private research centers.
L'archive ouverte pluridisciplinaire HAL, est destinée au dépôt et à la diffusion de documents scientifiques de niveau recherche, publiés ou non, émanant des établissements d'enseignement et de recherche français ou étrangers, des laboratoires publics ou privés. 


\title{
Integral sliding mode control of an extended Heisenberg system*
}

\author{
Michael Defoort ${ }^{1}$, Thierry Floquet ${ }^{2}$ \\ Wilfrid Perruquetti ${ }^{2}$ and Sergey V. Drakunov ${ }^{3}$
}

${ }^{1}$ Department of System Design Engineering, Keio University, Yokohama, Japan

${ }^{2}$ LAGIS UMR CNRS 8146, Ecole Centrale de Lille, BP 48, Cité Scientifique, 59651 Villeneuve-d'Ascq, FRANCE

\author{
${ }^{3}$ Embry-Riddle Aeronautical University, Department of Physical Sciences, \\ 600 S. Clyde Morris Blvd, Daytona Beach, FL 32114, USA.
}

\begin{abstract}
This paper deals with the practical robust stabilization and tracking problems of the perturbed multidimensional Heisenberg system with some additional integrators in the control input path. This objective is achieved by the use of variable structure control laws with an integral augmented sliding variable. This note shows how to select the integral sliding surface in such a way that the practical stabilization of the extended Heisenberg system is achieved in spite of the uncertainties and without loss of controllability. Experimental results on a wheeled mobile robot show the performance of the proposed controller for the practical stabilization and tracking problems.
\end{abstract}

Keywords: Heisenberg system, Sliding mode control, Higher order sliding mode, Wheeled mobile robot.

\section{Introduction}

The problem of stabilizing nonholonomic systems, i.e. systems subject to constraints that are often expressed in terms of nonintegrable linear velocity relationships, has received much attention (see [1] for an extended survey). It is because the control of nonholonomic systems arises in numerous applications, and also because those systems fail necessary conditions for the existence of smooth static state feedback that guarantees asymptotic stability of the equilibrium (see [2]). Thus, it is a challenging nonlinear control problem. Works on the stabilization problem for such systems have mainly focused on the design of time-varying or discontinuous feedback controllers. Thus, many control strategies such as smooth time-varying feedbacks [3], sinusoidal and polynomial controls [4], controls based on backstepping approaches [5], and nonsmooth feedbacks [6, 7, 8, 9] have been investigated.

\footnotetext{
${ }^{*}$ This work was partially supported by the JSPS Postdoctoral Fellowship and the FEDER (European Funds of Regional Development) under the ARCIR Robocoop and the AUTORIS-TAT T31 projects.
} 
An important class of such systems is the Heisenberg system, or nonholonomic integrator:

$$
\left\{\begin{array}{l}
\dot{x}_{1}=x_{2} u_{2}-x_{3} u_{1} \\
\dot{x}_{2}=u_{1} \\
\dot{x}_{3}=u_{2}
\end{array}\right.
$$

It belongs to the general class of driftless systems where the dimension of the control vector is smaller than that of the state vector. The problem of stabilizing this class of system is a nontrivial task since the linearized part is not controllable. In spite of its inherent difficulties, there are many practical reasons for focusing on this particular system since many mechanical systems like wheeled mobile robots, rolling knife or electromechanical systems such as current-fed induction motors are closely related to the nonholonomic integrator.

In many works about the stabilization of the Heisenberg system $[7,8,10]$ (or, in general, about the stabilization of nonholonomic systems $[11,12,13]$ ), the derived control laws are of discontinuous type and can lead to discontinuous velocities in practice. This difficulty can be overcome by adding cascade integrators in the path of the usual control inputs so that the discontinuous part of the control is embedded in higher time derivatives of the variables associated to the mechanical parts. Furthermore, applying a discontinuous control on electrical inputs appears relevant since the latter are most of time made of switching electrical devices. Stabilization or tracking of this extended Heisenberg system with additional integrators finds direct applications in the control of voltagefed induction motors, mobile robots for which the actuator dynamics are taken into account or Autonomous Underwater Vehicle (AUV).

In practical applications, systems operate under uncertainty conditions (modeling errors, external disturbances, etc.). Therefore, an important issue for a practical control design is the robustness consideration. Most of robust stabilization results and investigations focus on the stabilization of chained form systems with matched uncertainties [13] (perturbations that enter the state equation at the same point as the control input), with sensor noise and external disturbances $[12,14,15]$.

The discussed claim for robustness in nonlinear control can be effectively accomplished using sliding mode control. This discontinuous control technique consists in constraining the system motion along manifolds of reduced dimensionality in the state space and is applicable to a broad variety of practical applications (see the books $[16,17,18]$ for further details). Robustness properties against various kinds of uncertainties such as parameter perturbations, external disturbances and measurement errors can be guaranteed. Emel'yanov et al. [19] generalized the common sliding mode idea to higher order sliding modes. In [20], the generalization of sliding modes is given in term of semigroups. So far, only a few researchers have investigated the design of sliding mode controllers for Heisenberg system with uncertainties. A recent work proposed a first order sliding mode controller for the practical stabilization of the perturbed Heisenberg system [21]. However, high gains are needed in the design of the sliding variable. This fact could yield difficulties to tune the control parameters. Another formulation for the sliding variable, based on the integral sliding mode concept, will be introduced here.

The integral sliding mode design concept, introduced in [22], has two main properties: first it has no reaching phase (i.e. an initial period of time in which the system has not yet reached the sliding surface) and second, resulting from the first one, it ensures insensitivity of the desired trajectory with respect to uncertainties from the initial time instant. The basic idea of integral sliding mode control is to include an integral term in the sliding surface such that the system trajectories start on the sliding surface from the initial time instant. In [23], the robustness properties of the integral sliding mode controller are extended to unmatched vanishing uncertainties. An integral sliding mode controller, which need off-line initialization, is given in [24]. In [25], the authors combine integral sliding mode control with other robust techniques like the $H_{\infty}$ approach. This approach eliminates the effect of the matched perturbation without amplifying the unmatched one.

In this paper, another interesting property of integral sliding mode control is highlighted. Indeed, this technique will be shown to be useful to avoid singularities in the matrix of the input channel. Since the system is constrained to evolve on the integral sliding surface from the initial 
time instant, the possible singularities in the control during the transient are avoided provided the sliding surface is suitably chosen. After designing the integral sliding surface, two, easily implementable, controllers are proposed. The first one is based on first order sliding mode and rejects the effect of matched uncertainties. Then, generalizing the concept of integral sliding mode, a second order integral sliding mode controller is designed to reject the effect of a larger class of uncertainties.

The paper is organized as follows. The problem statement and some illustrative examples are reported in Section 2. Then, two integral sliding mode control laws are designed in Section 3. In Section 4, the proposed control methodology is applied to solve the tracking problem. Finally, experimental results for the practical stabilization and tracking of a wheeled mobile robot are given.

\section{Problem statement and motivations}

\subsection{Motivating physical examples}

Induction motor: The link between the current-fed induction motor and the double integrator system has been highlighted in [26]. The Park model of the voltage-fed induction motor in the $(\alpha, \beta)$ frame (see e.g. [27]), under the hypothesis of magnetic circuit with linear characteristics, is given by:

$$
\left\{\begin{array}{l}
\dot{z}=\gamma_{1} \mathbf{I}^{T} J \boldsymbol{\Phi}-\gamma_{2} z-\gamma_{3} C_{l} \\
\dot{\boldsymbol{\Phi}}=\gamma_{4} \mathbf{I}-\gamma_{5} \boldsymbol{\Phi}+\gamma_{6} z J \boldsymbol{\Phi}+\Delta_{1}(\mathbf{I}, \boldsymbol{\Phi}) \\
\dot{\mathbf{I}}=\gamma_{7} \mathbf{U}-\gamma_{8} \mathbf{I}+\gamma_{9} \boldsymbol{\Phi}-\gamma_{10} z J \boldsymbol{\Phi}+\Delta_{2}(\mathbf{I}, \boldsymbol{\Phi})
\end{array}\right.
$$

where $z \in \mathbb{R}$ is the rotor speed, $\mathbf{I}=\left[i_{s \alpha}, i_{s \beta}\right]^{T} \in \mathbb{R}^{2}$ are the stator currents and $\boldsymbol{\Phi}=\left[\phi_{r \alpha}, \phi_{r \beta}\right]^{T} \in$ $\mathbb{R}^{2}$ are the rotor fluxes. The control inputs are the two stator voltages $\mathbf{U}=\left[V_{s \alpha}, V_{s \beta}\right]^{T} . J=$ $\left[\begin{array}{cc}0 & -1 \\ 1 & 0\end{array}\right]$ is a skew symmetric matrix. The different parameters $\gamma_{i}$ involved in (2) depend on the considered machine (number of pole pairs, moment of inertia, viscous coefficient, rotor and stator winding resistances, inductances). Particularly, $\gamma_{4}, \gamma_{5}, \gamma_{8}$ and $\gamma_{9}$ depend on the rotor resistance whose value can drastically change while operating due to rotor heating. $\Delta_{1}, \Delta_{2}$ stand for some unknown parametric uncertainties in the electrical subsystem. $C_{l}$ denotes the load torque and is generally unknown.

Wheeled mobile robot: It is known, that a third order nonholonomic systems of the form $\dot{\mathbf{x}}=B(\mathbf{x}) \mathbf{u}$ can be locally written in a form similar to the so-called Heisenberg system [29, 2]. For example, the link between the wheeled mobile robot and the double integrator system has been highlighted in [28]. Indeed, consider a unicycle-type mobile robot, see Fig. 1, with two driving wheels mounted on the same axis and independently controlled by two actuators (DC motors). This robot is fully described by a three dimensional vector of generalized coordinates q constituted by the coordinates $(x, y)$ of the midpoint between the two driving wheels and by the orientation angle $\theta$ with respect to a fixed frame:

$$
\mathbf{q}=[x, y, \theta]^{T}
$$

Under the pure rolling and non slipping condition [30], the ideal kinematic equations are:

$$
\dot{\mathbf{q}}=\left[\begin{array}{cc}
\cos \theta & 0 \\
\sin \theta & 0 \\
0 & 1
\end{array}\right]\left[\begin{array}{c}
w_{l} \\
w_{a}
\end{array}\right]
$$

where $w_{l}$ and $w_{a}$ are the linear and angular velocities, respectively. 


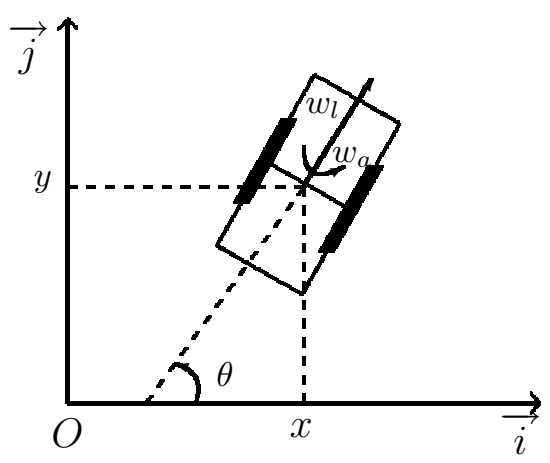

Figure 1: Unicycle-type mobile robot

In order to express the unicycle type model in the Heisenberg form, the following global invertible transformation is defined (see [28] for further details):

$$
\left[z, \mathbf{X}^{T}\right]^{T}=T(\theta) \quad[x, y, \theta]^{T}
$$

where the matrix $T(\theta) \in \mathbb{R}^{3 \times 3}$ is defined as:

$$
T(\theta)=\left[\begin{array}{ccc}
\theta \cos \theta-2 \sin \theta & \theta \sin \theta+2 \cos \theta & 0 \\
0 & 0 & 1 \\
\cos \theta & \sin \theta & 0
\end{array}\right]
$$

and where $z \in \mathbb{R}$ and $\mathbf{X}=\left[x_{1}, x_{2}\right] \in \mathbb{R}^{2}$ are the auxiliary tracking errors variables.

Differentiating $z$ and $\mathbf{X}$ with respect to time and using equations (4)-(6) yield the following differential equations:

$$
\begin{aligned}
\dot{z}= & (-\theta \sin \theta-\cos \theta) w_{a} x+(\theta \cos \theta-2 \sin \theta) w_{l} \cos \theta \\
& +(\theta \cos \theta-\sin \theta) w_{a} y+(\theta \sin \theta+2 \cos \theta) w_{l} \sin \theta \\
\dot{z}= & (-\cos \theta-\theta \sin \theta) w_{a} x+\theta w_{l}+(-\sin (\theta)+\theta \cos \theta) w_{a} y \\
\dot{z}= & \theta\left(w_{l}-w_{a}(x \sin \theta-y \cos \theta)\right)-(x \cos \theta+y \sin \theta) w_{a}
\end{aligned}
$$

and

$$
\dot{\mathbf{X}}=\left[\begin{array}{c}
w_{a} \\
\cos \theta w_{l} \cos \theta+\sin \theta w_{l} \sin \theta-w_{a}(x \sin \theta-y \cos \theta)
\end{array}\right]=\left[\begin{array}{c}
w_{a} \\
w_{l}-w_{a}(x \sin \theta-y \cos \theta)
\end{array}\right]
$$

Hence, the tracking error dynamics can be expressed in terms of the auxiliary variables defined in (5) as follows:

$$
\left\{\begin{array}{l}
\dot{z}=\mathbf{Y}^{T} J \mathbf{X} \\
\dot{\mathbf{X}}=\mathbf{Y}
\end{array}\right.
$$

where $J$ is a constant, skew symmetric matrix defined as:

$$
J=\left[\begin{array}{cc}
0 & -1 \\
1 & 0
\end{array}\right]
$$

The auxiliary variable $\mathbf{Y}=\left[y_{1}, y_{2}\right]^{T} \in \mathbb{R}^{2}$ introduced in (7) is defined in terms of the unicycle-type mobile robot position and orientation, linear and angular velocities as follows:

$$
\left\{\begin{array}{l}
y_{1}=w_{a} \\
y_{2}=w_{l}-w_{a}(x \sin \theta-y \cos \theta)
\end{array}\right.
$$


Since the proposed control law is based on sliding mode control which is of discontinuous type and may lead to discontinuous velocities in practice, one can take into account the dynamical model of the mobile robot such that the discontinuous part of the control is embedded in the higher time derivatives of the mechanical parts. From this point of view, the problem becomes to solve the stabilization and the tracking of the system defined by

$$
\left\{\begin{array}{l}
\dot{z}=\mathbf{Y}^{T} J \mathbf{X} \\
\dot{\mathbf{X}}=\mathbf{Y} \\
\dot{\mathbf{Y}}=\mathbf{U}
\end{array}\right.
$$

where $\mathbf{U} \in \mathbb{R}^{2}$ is the new control input.

\subsection{General problem}

Motivated by the above mechanical and electromechanical examples, it is of practical interest to study the following $(2 n+1)^{t h}$ order nonlinear system $(n \geq 2)$, which is a class of extended Heisenberg system with additional integrators in the control input path:

$$
\begin{aligned}
\dot{z} & =\mathbf{Y}^{T} J_{1}(\boldsymbol{\Sigma}, t) \mathbf{X}+\delta_{1}(\boldsymbol{\Sigma}, t) \\
\dot{\mathbf{X}} & =\mathbf{Y}+J_{2}(\boldsymbol{\Sigma}, t) \mathbf{X}+\delta_{2}(\boldsymbol{\Sigma}, t) \\
\dot{\mathbf{Y}} & =\mathbf{U}+\delta_{3}(\boldsymbol{\Xi}, t)
\end{aligned}
$$

where the state vector is $\boldsymbol{\Xi}=\left[z, \mathbf{X}^{T}, \mathbf{Y}^{T}\right]^{T} \in \mathbb{R}^{2 n+1}, z \in \mathbb{R}, \mathbf{X}, \mathbf{Y} \in \mathbb{R}^{n}$, where the control input is $\mathbf{U} \in \mathbb{R}^{n}$ and where $\boldsymbol{\Sigma}=\left[z, \mathbf{X}^{T}\right]^{T} \in \mathbb{R}^{n+1}$ is the output to be controlled. $\delta_{i}, i=1,2,3$, represents parametric uncertainties or disturbances and is assumed to be a sufficiently smooth function such that:

$$
\left\{\begin{array}{l}
\left\|\delta_{i}(\boldsymbol{\Sigma}, t)\right\| \leq \bar{\delta}_{i}(\boldsymbol{\Sigma}) \\
\left\|\dot{\delta}_{i}(\boldsymbol{\Sigma}, t)\right\| \leq \bar{\delta}_{i}^{\prime}(\boldsymbol{\Sigma})
\end{array} \quad i=1,2\right.
$$

and

$$
\left\|\delta_{3}(\boldsymbol{\Xi}, t)\right\| \leq \bar{\delta}_{3}(\boldsymbol{\Xi})
$$

where $\bar{\delta}_{i}$ and $\bar{\delta}_{i}^{\prime}$ are known nonnegative functions. It is worth stressing, that if $\delta_{1}(\boldsymbol{\Sigma}, t) \neq 0$ and $\delta_{2}(\boldsymbol{\Sigma}, t) \neq 0$, the disturbances are not matched and are not assumed to be vanishing.

$J_{1}(\boldsymbol{\Sigma}, t)$ and $J_{2}(\boldsymbol{\Sigma}, t)$ are $n \times n$ skew symmetric matrices (i.e. such that $J_{i}^{T}=-J_{i}$ ), which can be state and time dependent. It is assumed that $J_{1}$ is nonsingular. Regarding $J_{2}$, no other than the skew symmetry condition is required, but, of course, this function as well as all others in the right hand side of (10)-(12) should guarantee existence and uniqueness of the solution on the infinite time interval.

Note that on the opposite to the standard Heisenberg system, equations (10)-(12) include uncertainties. The presence of the uncertainties in the right-hand side makes the class of systems different from the one considered in [12, 15]. Furthermore, in these works (and also in [11, 13]), discontinuous controllers were designed leading to the stabilization of chained systems, with or without dynamical extension. However, the system (10)-(12) cannot always be transformed in the chained form with additional integrators.

The system (10)-(12), if undisturbed, can be seen as the interconnection of two subsystems: a $2 n$ order one represented by a chain of two $n$-dimensional integrators with possible drift terms, and a scalar nonlinear system whose dynamics is entirely driven by the subsystem (10).

The problems considered here is to find practical stabilizing and tracking control laws for the state vector $\boldsymbol{\Sigma}$ in spite of the presence of parameter uncertainties and disturbances. Here 'practical stability' has to be understood in the following sense: $z$ converges to zero while $X$ tends to a value that can be made arbitrarily small selecting sufficiently large controller gains (that depends on the size of the perturbations). 


\section{Practical stabilization of the extended Heisenberg system}

Here, the control laws will be designed using sliding mode techniques. The aim of sliding mode control is, by means of a discontinuous control, to robustly constrain the system to reach and stay, after a finite time, on a sliding surface $\mathcal{S}^{r}$ where the resulting behavior has some prescribed dynamics. The sliding surface is defined by the vanishing of a corresponding sliding vector $s$ and its successive time derivatives up to a certain order, i.e. the $r^{\text {th }}$ order sliding set:

$$
\mathcal{S}^{r}=\left\{(t, \boldsymbol{\Xi}): \boldsymbol{s}=\dot{\boldsymbol{s}}=\ldots=\boldsymbol{s}^{(r-1)}=0\right\}
$$

A control law leading to such a behavior is called a $r^{\text {th }}$ order ideal sliding mode algorithm with respect to $s$. Higher order sliding modes, that are characterized by a discontinuous control acting on the $r^{t h}, r>1$, time derivatives of the sliding vector (instead of the first time derivative in classical sliding mode, $r=1$ ), can reduce the chattering phenomenon while preserving the robustness properties. Indeed, assume that the system has relative degree ${ }^{1} p$ with respect to the sliding vector $s$. If the sliding mode order $r$ is chosen such that $p \leqslant r-1$, the discontinuous algorithm generating the $r^{\text {th }}$ order sliding mode is applied to the $r-p$ time derivative of the control input and thus the actual input of the system is continuous. Furthermore, they guarantee better convergence accuracy (see e.g. [32, 33] for a survey).

The robust sliding mode control of the extended Heisenberg system is designed in two steps:

1. the selection of a suitable integral sliding vector s such that, while sliding, the control objective is fulfilled,

2. the design of the corresponding control input that constrains the system trajectories to evolve, after a finite time and in spite of the uncertainties, on the sliding surface $\mathcal{S}^{r}$.

REMARK 1 One of the main difficulty is the fact that the stabilization of $\mathbf{X}$ leads to zeroing righthand side of (10) and therefore, the variable z cannot be steered to zero anymore. This simple observation implies that $\mathbf{X}$ must not vanish before $z$ in order to stabilize system (10)-(12). Therefore, it is critical to select an appropriate sliding surface.

Assumption 1 It is assumed that the initial condition on the variable $\mathbf{X}$ is not equal to zero, i.e.

$$
\|\mathbf{X}(0)\|^{2}>\epsilon
$$

with $1 \gg \epsilon>0$.

REMARK 2 If $\|\mathbf{X}(0)\|=0$, then, before applying the controller which will be proposed hereafter, an initialization step is needed in order to go far enough from the manifold $\|\mathbf{X}\|=0$.

\subsection{System with matched uncertainties}

It is assumed in this part that $\delta_{1}(\boldsymbol{\Sigma}, t)=\delta_{2}(\boldsymbol{\Sigma}, t)=0$. In this case, it is shown here that a first order integral sliding mode control can be designed such that the system (10)-(12) is practically stable.

\subsubsection{Integral augmented sliding vector}

Since $\mathbf{X}$ must not vanish before $z$, it appears interesting to define the following sliding vector $\mathbf{s}=\left[s_{1}, s_{2}\right]^{T} \in \mathbb{R}^{2}$ as:

$$
\left\{\begin{array}{l}
s_{1}(t)=\dot{z}(t)+k_{1} z(t)+k_{2} a_{1}(t) \\
s_{2}(t)=\dot{\psi}(t)+k_{3} \psi(t)+k_{4} a_{2}(t)
\end{array}\right.
$$

\footnotetext{
${ }^{1}$ i.e the number of times $\boldsymbol{s}$ have to be differentiated before the control input explicitly appears (see [31] p. 220 for further details about the definition of relative degree).
} 
Since $\delta_{1}(\boldsymbol{\Sigma}, t)=\delta_{2}(\boldsymbol{\Sigma}, t)=0$, it can also be expressed as:

$$
\left\{\begin{array}{l}
s_{1}=\mathbf{Y}^{T} J_{1} \mathbf{X}+k_{1} z+k_{2} a_{1} \\
s_{2}=\mathbf{X}^{T}\left(\mathbf{Y}+J_{2} \mathbf{X}\right)-\frac{\partial \varphi(z)}{\partial z} \mathbf{Y}^{T} J_{1} \mathbf{X}+k_{3} \psi+k_{4} a_{2}
\end{array}\right.
$$

where

- the variable $\psi$ is defined as:

$$
\psi=\frac{1}{2} \mathbf{X}^{T} \mathbf{X}-\varphi(z)-\frac{1}{2} \epsilon
$$

- $k_{1}$ and $k_{2}$ ( $k_{3}$ and $k_{4}$, respectively) are strictly positive constants which define the convergence rate of $z$ ( $\psi$, respectively),

- the variables $a_{1}$ and $a_{2}$ induce the integral term and provide one more degree of freedom in the sliding vector design. Their dynamics fulfill the following differential equations:

$$
\left\{\begin{array}{l}
\dot{a}_{1}(t)=z(t) \\
\dot{a}_{2}(t)=\psi(t) \\
a_{1}(0)=-\frac{1}{k_{2}}\left(\mathbf{Y}^{T} J_{1} \mathbf{X}+k_{1} z\right)(0) \\
a_{2}(0)=-\frac{1}{k_{4}}\left(\mathbf{X}^{T}\left(\mathbf{Y}+J_{2} \mathbf{X}\right)-\frac{\partial \varphi(z)}{\partial z} \mathbf{Y}^{T} J_{1} \mathbf{X}+k_{3} \psi\right)(0)
\end{array}\right.
$$

The initial conditions $a_{1}(0)$ and $a_{2}(0)$ are determined such that the sliding vector always satisfies $\mathbf{s}(0)=0$ and imply the knowledge of initial conditions $\mathbf{X}(0), \mathbf{Y}(0)$ and $z(0)$. Hence, the controlled system slides on the sliding surface $\mathcal{S}^{1}=\{\mathbf{s}=0\}$ from the initial time instant without any reaching phase.

- the function $\varphi: \mathbb{R} \rightarrow \mathbb{R}^{+}$is a twice differentiable positive definite function, i.e.

$$
\left\{\begin{array}{lll}
\varphi(z)>0 & \text { if } & z \neq 0 \\
\varphi(z)=0 & \text { if } & z=0
\end{array}\right.
$$

It is chosen as a radially unbounded convex function and such that the condition $\psi(0) \geq 0$ is satisfied, that is to say:

$$
\frac{\|\mathbf{X}(0)\|^{2}-\epsilon}{2} \geq \varphi(z(0))
$$

REMARK 3 If Assumption 1 is satisfied, one can always design a positive definite function $\varphi$ satisfying the above conditions. Indeed, one can choose for instance: $\varphi(z)=c z^{2 m}$ where $c \in \mathbb{R}^{+}$and $m \in \mathbb{N}$ are suitable constants. Note that this function implies a degree of correspondence between the convergence rates of $\|\mathbf{X}\|$ and $z$.

Lemma 3.1 If Assumption 1 is satisfied, there exist positive constants $k_{i}$ and a positive definite function $\varphi(z)$ such that, in sliding mode, the system (10)-(12) is practically stable.

Proof. It is assumed that a first order sliding mode is generated on the surface given by the equality $\mathbf{s}=0$. Therefore, in sliding mode, since $\dot{\mathbf{s}}=0, z$ and $\psi$ are constrained to fulfill the following differential equations:

$$
\left\{\begin{array}{l}
\ddot{z}=-k_{1} \dot{z}-k_{2} z \\
\ddot{\psi}=-k_{3} \dot{\psi}-k_{4} \psi
\end{array}\right.
$$

The positive definite function $\varphi(z)$ is chosen such that $\psi(0) \geq 0$, i.e condition (20) holds. The positive constants $k_{1}, k_{2}, k_{3}, k_{4}$ are selected straightforwardly using pole assignment technique such that, in sliding mode, $z$ and $\psi$ are exponentially stable and $\psi(t) \geq 0$ for all $t \geq 0$. Therefore, if the discontinuous control law $\mathbf{U}$ constrains the system trajectories to evolve on the sliding surface $\{\mathbf{s}=0\}$ from the initial time instant, one gets the desired properties: 
- $\mathbf{X}^{T} \mathbf{X} \geq 2 \varphi(z)+\epsilon \Longrightarrow \mathbf{X}^{T} \mathbf{X}>0$ for all $t \geq 0$.

- In sliding mode, the system (10)-(12) is practically stable. Indeed, since $z$ and $\psi$ converge exponentially toward zero, $\|\mathbf{X}\|^{2}$ converges exponentially toward $\epsilon$ since $\varphi$ is positive definite.

\subsubsection{Design of a first order sliding mode controller}

Here, the control input $\mathbf{U}$ is designed such that a sliding mode occurs on $\mathbf{s}=0$ from the initial time instant. Note that the relative degree of the system (10)-(12) with respect to the sliding vector is $[1,1]^{T}$. Indeed, the time derivative of $\mathbf{s}$ along the trajectories of (10)-(12) is given by:

$$
\dot{\mathbf{s}}=M(\boldsymbol{\Xi}) \mathbf{U}+\Gamma(\boldsymbol{\Xi})+\Theta(\boldsymbol{\Xi})
$$

where

$$
\begin{gathered}
M(\boldsymbol{\Xi})=\left[\begin{array}{c}
-\mathbf{X}^{T} J_{1} \\
\mathbf{X}^{T}+\frac{\partial \varphi(z)}{\partial z} \mathbf{X}^{T} J_{1}
\end{array}\right] \\
\Gamma(\boldsymbol{\Xi})=\left[\begin{array}{l}
\mathbf{Y}^{T} J_{1} J_{2} \mathbf{X}+\mathbf{Y}^{T} \dot{J}_{1} \mathbf{X}+k_{1} \mathbf{Y}^{T} J_{1} \mathbf{X}+k_{2} z \\
\mathbf{Y}^{T}\left(\mathbf{Y}+J_{2} \mathbf{X}\right)+\mathbf{X}^{T} \dot{J}_{2} \mathbf{X}-\frac{\partial^{2} \varphi(z)}{\partial z^{2}}\left(\mathbf{Y}^{T} J_{1} \mathbf{X}\right)^{2}-\frac{\partial \varphi(z)}{\partial z}\left(\mathbf{Y}^{T} J_{1} J_{2} \mathbf{X}+\mathbf{Y}^{T} \dot{J}_{1} \mathbf{X}\right) \\
+k_{3} \mathbf{X}^{T}\left(\mathbf{Y}+J_{2} \mathbf{X}\right)-k_{3} \frac{\partial \varphi(z)}{\partial z} \mathbf{Y}^{T} J_{1} \mathbf{X}+k_{4} \psi
\end{array}\right]
\end{gathered}
$$

and

$$
\Theta(\boldsymbol{\Xi})=\left[\begin{array}{c}
-\mathbf{X}^{T} J_{1} \delta_{3}(\boldsymbol{\Xi}) \\
\mathbf{X}^{T}\left(\frac{\partial \varphi(z)}{\partial z} J_{1} \delta_{3}(\boldsymbol{\Xi})+\delta_{3}(\boldsymbol{\Xi})\right)
\end{array}\right]
$$

The system is strongly coupled by the matrix $M(\boldsymbol{\Xi}) \in \mathbb{R}^{2 \times n}$. In order to circumvent this problem, let us define the static feedback:

$$
\mathbf{U}=\left[\begin{array}{ll}
J_{1} \mathbf{X} & \mathbf{X}-\frac{\partial \varphi(z)}{\partial z} J_{1} \mathbf{X}
\end{array}\right] \mathbf{W}
$$

where $\mathbf{W} \in \mathbb{R}^{2}$. Equation (22) becomes:

$$
\dot{\mathbf{s}}=N(\boldsymbol{\Xi}) \mathbf{W}+\Gamma(\boldsymbol{\Xi})+\Theta(\boldsymbol{\Xi})
$$

with

$$
\begin{aligned}
N(\boldsymbol{\Xi}) & =\left[\begin{array}{c}
-\mathbf{X}^{T} J_{1} \\
\mathbf{X}^{T}+\frac{\partial \varphi(z)}{\partial z} \mathbf{X}^{T} J_{1}
\end{array}\right]\left[\begin{array}{cc}
J_{1} \mathbf{X} & \mathbf{X}-\frac{\partial \varphi(z)}{\partial z} J_{1} \mathbf{X}
\end{array}\right] \\
& =\left[\begin{array}{cc}
\left\|J_{1} \mathbf{X}\right\|^{2} & -\frac{\partial \varphi(z)}{\partial z}\left\|J_{1} \mathbf{X}\right\|^{2} \\
-\frac{\partial \varphi(z)}{\partial z}\left\|J_{1} \mathbf{X}\right\|^{2} & \|\mathbf{X}\|^{2}+\left(\frac{\partial \varphi(z)}{\partial z}\right)^{2}\left\|J_{1} \mathbf{X}\right\|^{2}
\end{array}\right]
\end{aligned}
$$

REMARK 4 Integral sliding mode control laws are usually designed to be robust with respect to uncertainties from the initial time instant. Here, another interesting application of integral sliding mode is highlighted: it is possible to avoid the control singularities that could appear during the reaching phase with classical sliding mode controls. Indeed: $\operatorname{det} N(\boldsymbol{\Xi})=\left\|J_{1} \mathbf{X}\right\|^{2}\|\mathbf{X}\|^{2}$. Thus, since $J_{1}$ is a nonsingular matrix, the decoupling matrix $N(\boldsymbol{\Xi})$ is always invertible except on the manifold $\mathbf{X}^{T} \mathbf{X}=0$. According to Lemma 3.1, $\mathbf{X}^{T} \mathbf{X}>0$ for all $t \geq 0$ when $\mathbf{s}=0$. As the transient process is removed, control singularities are avoided. 
The control law $\mathbf{W}$ can be designed in the following form:

$$
\mathbf{W}=\mathbf{W}^{0}+\Delta \mathbf{W}
$$

The control $\mathbf{W}^{0}$ is directly determined according to the choice of the integral sliding vector:

$$
\mathbf{W}^{0}=-N(\boldsymbol{\Xi})^{-1} \Gamma(\boldsymbol{\Xi})
$$

$\Delta \mathbf{W}$ represents the discontinuous part of the control and is defined to enforce a sliding motion on $\mathbf{s}=0$, in finite time and in spite of the uncertainties:

$$
\Delta \mathbf{W}=-N(\boldsymbol{\Xi})^{-1} K(\boldsymbol{\Xi}) \operatorname{Sign}(\mathbf{s})
$$

where

and the gain satisfies

$$
\operatorname{Sign}(\mathbf{s})=\left[\operatorname{sign}\left(s_{1}\right), \operatorname{sign}\left(s_{2}\right)\right]^{T}
$$

$$
K(\boldsymbol{\Xi})>\|\Theta(\boldsymbol{\Xi})\|+\eta
$$

with $\eta>0$.

Theorem 3.2 Consider the system (10)-(12) with $\delta_{1}(\boldsymbol{\Sigma}, t)=\delta_{2}(\boldsymbol{\Sigma}, t)=0$ and assume that there exist a constant $1 \gg \epsilon>0$ such that $\|\mathbf{X}(0)\|^{2}>\epsilon$, positive constants $k_{i}(i=1, \ldots, 4)$ and a twice differentiable positive definite function $\varphi(z)$ such that condition (20) is verified.

Then, using the controller defined in equations (26)-(28), with the sliding vector (17), the practical stabilization of the state $\left[z, \mathbf{X}^{T}\right]^{T}$ is ensured.

Proof. In order to demonstrate that the system trajectories converge in finite time onto the sliding surface $\{\mathbf{s}=0\}$, let us choose the following Lyapunov function:

$$
V=\frac{1}{2} \mathbf{s}^{T} \mathbf{s}
$$

The time derivative of $V$ along the system trajectories is expressed as:

$$
\begin{aligned}
\dot{V} & =\mathbf{s}^{T}(N(\boldsymbol{\Xi}) \mathbf{W}+\Gamma(\boldsymbol{\Xi})+\Theta(\boldsymbol{\Xi})) \\
& =\mathbf{s}^{T}(-K(\boldsymbol{\Xi}) \operatorname{Sign}(\mathbf{s})+\Theta(\boldsymbol{\Xi}))
\end{aligned}
$$

Inequality (29) implies that

$$
\dot{V} \leq-\eta \sqrt{V}
$$

Hence, the system trajectories evolve on the surface $\{\mathbf{s}=0\}$ in finite time and remains there in spite of the uncertainties. Using the control input with the proposed sliding vector, the Heisenberg system, considered in this paper, can be controlled in the sliding mode from the initial time instant since $\mathbf{s}(0)=0$. Thus, the discontinuous control term maintains the system on the sliding surface by rejecting the effect of the uncertainties for $t \geq 0$ and avoids control singularities (i.e. $\|\mathbf{X}\| \neq 0$ ). Due to lemma 3.1, one obtains the practical stabilization of the state.

\subsection{System with unmatched uncertainties}

In this part, arbitrary uncertainties are considered. The design is still based on integral sliding mode controller. However, the integral sliding vector (17) cannot be considered in the controller design since $\dot{z}$ and $\dot{\mathbf{X}}$ are subject to perturbations. In the following, a second order sliding mode control law is proposed in order to circumvent this difficulty. The control objective is to generate a second order sliding mode onto a sliding surface appropriately chosen, that is to say, to constrain the system trajectories to evolve from the initial time instant on

$$
\mathcal{S}^{2}=\{(t, \boldsymbol{\Xi}): \boldsymbol{\sigma}=\dot{\boldsymbol{\sigma}}=0\}
$$

The proposed solution is based on a generalization of the integral sliding mode principle to second order sliding mode controllers. 


\subsubsection{Integral augmented sliding vector}

In this part, it is assumed that $\dot{z}(0)$ and $\dot{\mathbf{X}}(0)$ are known.

REMARK 5 The condition of the knowledge of $\dot{z}(0)$ and $\dot{\mathbf{X}}(0)$ is not restrictive. Indeed, before applying the proposed controller hereafter, one can design a robust finite time exact differentiator in order to obtain the initial conditions. For further details about this technique, one can refer to [34].

Let us define the sliding vector $\boldsymbol{\sigma}=\left[\sigma_{1}, \sigma_{2}\right]^{T}$ as:

$$
\left\{\begin{array}{l}
\sigma_{1}(t)=z(t)+k_{1} a_{1}(t)+k_{2} b_{1}(t) \\
\sigma_{2}(t)=\psi(t)+k_{3} a_{2}(t)+k_{4} b_{2}(t)
\end{array}\right.
$$

where

- the variable $\psi$ is defined as in (18),

- $k_{1}$ and $k_{2}$ ( $k_{3}$ and $k_{4}$ respectively) are strictly positive constants which define the convergence rate of $z$ ( $\psi$ respectively),

- the variables $a_{1}, a_{2}, b_{1}$ and $b_{2}$ still induce the integral term. Their dynamics fulfill the following differential equations:

$$
\left\{\begin{array}{l}
\dot{a}_{1}(t)=z(t) \\
\dot{b}_{1}(t)=a_{1}(t) \\
\dot{a}_{2}(t)=\psi(t) \\
\dot{b}_{2}(t)=a_{2}(t)
\end{array}\right.
$$

with

$$
\left\{\begin{array}{l}
a_{1}(0)=-\frac{1}{k_{2}}\left(\dot{z}+k_{1} z\right)(0) \\
b_{1}(0)=-\frac{1}{k_{2}}\left(z+k_{1} a_{1}\right)(0) \\
a_{2}(0)=-\frac{1}{k_{4}}\left(\mathbf{X}^{T} \dot{\mathbf{X}}-\frac{\partial \varphi(z)}{\partial z} \dot{z}+k_{3} \psi\right)(0) \\
b_{2}(0)=-\frac{1}{k_{4}}\left(\psi+k_{3} a_{2}\right)(0)
\end{array}\right.
$$

The initial conditions are determined such that the sliding vector always satisfies $\dot{\boldsymbol{\sigma}}(0)=$ $\boldsymbol{\sigma}(0)=0$. Hence, the controlled system slides on $\mathcal{S}^{2}$ from the initial time instant without any reaching phase.

- the function $\varphi: \mathbb{R} \rightarrow \mathbb{R}^{+}$is a twice differentiable positive definite function. It is chosen as a radially unbounded convex function such that condition (20) holds.

Lemma 3.3 If Assumption 1 is satisfied, there exist positive constants $k_{i}$ and a positive definite function $\varphi(z)$ such that, in sliding mode, the system (10)-(12) is practically stable.

Proof. The proof is similar to lemma 3.1. It is assumed that a second order sliding mode is generated on the surface given by the equalities: $\boldsymbol{\sigma}=\boldsymbol{\sigma}=0$. Therefore, in sliding mode, $z$ and $\psi$ are constrained to fulfill the differential equations (21). The positive constants $k_{1}, k_{2}, k_{3}, k_{4}$ are selected straightforwardly using pole assignment technique such that, in sliding mode, $z$ and $\psi$ are exponentially stable and $\psi(t) \geq 0$ (because (20) holds). Therefore, if the discontinuous control law $\mathbf{U}$ constrains the system trajectories to evolve on the sliding surface $\{\boldsymbol{\sigma}=\dot{\boldsymbol{\sigma}}=0\}$ from the initial time instant, one gets the desired properties:

- $\mathbf{X}^{T} \mathbf{X}>0$ for all $t \geq 0$.

- In sliding mode, the system (10)-(12) is practically stable. 


\subsubsection{Design of a second order sliding mode controller}

Here, the control input $\mathbf{U}$ is designed such that a sliding mode occurs on $\{\boldsymbol{\sigma}=\dot{\boldsymbol{\sigma}}=0\}$ from the initial time instant. Note that the relative degree of the system (10)-(12) with respect to the sliding vector $\boldsymbol{\sigma}$ is $[2,2]^{T}$. Indeed, the second time derivative of $\boldsymbol{\sigma}$ along the trajectories of (10)-(12) is given by:

$$
\ddot{\boldsymbol{\sigma}}=M(\boldsymbol{\Xi}) \mathbf{U}+\Gamma(\boldsymbol{\Xi})+\widetilde{\Theta}(\boldsymbol{\Xi})
$$

where

$$
\widetilde{\Theta}(\boldsymbol{\Xi})=\left[\begin{array}{l}
\mathbf{Y}^{T} J_{1} \delta_{2}-\mathbf{X}^{T} J_{1} \delta_{3}+\dot{\delta}_{1}+k_{1} \delta_{1} \\
\delta_{2}^{T}\left[2 \mathbf{Y}+J_{2} \mathbf{X}+\delta_{2}+\frac{\partial \varphi(z)}{\partial z} J_{1} \mathbf{Y}\right]+\mathbf{X}^{T}\left(\frac{\partial \varphi(z)}{\partial z} J_{1} \delta_{3}+\delta_{3}+\dot{\delta}_{2}\right) \\
-\frac{\partial^{2} \varphi(z)}{\partial z^{2}} \delta_{1}\left[2 \mathbf{Y}^{T} J_{1} \mathbf{X}+\delta_{1}\right]-\frac{\partial \varphi(z)}{\partial z} \dot{\delta}_{1}+k_{3} \mathbf{X}^{T} \delta_{2}-k_{3} \frac{\partial \varphi(z)}{\partial z} \delta_{1}
\end{array}\right]
$$

As in the previous section, the static feedback given by equation (23) enables to transform the system (33) into:

$$
\ddot{\boldsymbol{\sigma}}=N(\boldsymbol{\Xi}) \widetilde{\mathbf{W}}+\Gamma(\boldsymbol{\Xi})+\widetilde{\Theta}(\boldsymbol{\Xi}) .
$$

In order to design second order sliding mode algorithms, the following assumption must hold (see for instance [32]). It is assumed that there exist positive constants $\sigma_{0}$ and $C_{0}$ such that $\forall \boldsymbol{\Xi} \in \mathbb{R}^{2 n+1}$ and $\|\boldsymbol{\sigma}(\Xi)\|<\sigma_{0}$, the system satisfies the following condition:

$$
\|\widetilde{\Theta}(\boldsymbol{\Xi})\|<C_{0}
$$

One can note that this assumption is relatively not restrictive since it is satisfied if $\widetilde{\Theta}$ is continuous on a compact set.

THEOREM 3.4 Consider the system (10)-(12) and assume that there exist a constant $1 \gg \epsilon>0$ such that $\|\mathbf{X}(0)\|^{2}>\epsilon$, positive constants $k_{i}(i=1, \ldots, 4)$ and a twice differentiable positive definite function $\varphi(z)$ such that condition (20) is satisfied.

Let us define the sliding mode controller:

$$
\widetilde{\mathbf{W}}=\widetilde{\mathbf{W}}^{0}+\Delta \widetilde{\mathbf{W}}
$$

where

$$
\widetilde{\mathbf{W}}^{0}=-N(\boldsymbol{\Xi})^{-1} \Gamma(\boldsymbol{\Xi})
$$

and

$$
\Delta \widetilde{\mathbf{W}}=\left[\Delta \widetilde{W}_{1}, \Delta \widetilde{W}_{2}\right]^{T}
$$

with, for $j=1,2$

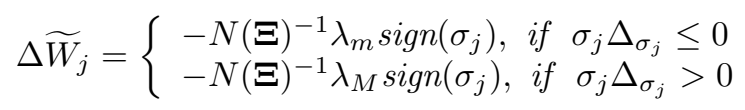

and

$$
\Delta_{\sigma_{j}}=\left\{\begin{array}{lll}
0, & \text { if } & k=0 \\
\sigma_{j}(k \tau)-\sigma_{j}((k-1) \tau), & \text { if } & k \geq 1
\end{array}\right.
$$

where $\tau$ is the sampling period. The control gains $\lambda_{m}$ and $\lambda_{M}$ satisfy the following conditions:

$$
\begin{aligned}
& \lambda_{m}>C_{0} \\
& \lambda_{M}>\lambda_{m}+2 C_{0}
\end{aligned}
$$

Then, the practical stabilization of the state $\left[z, \mathbf{X}^{T}\right]^{T}$ is ensured. 
Proof. The sampled twisting algorithm used in equations (37)-(38) provides good robustness properties. It does not require the knowledge of the time derivative of the sliding variable $\boldsymbol{\sigma}$ and takes into account some practical constraints such as the sampling of the measurement and the control. It can be shown that this controller ensures a finite time convergence of the trajectories onto the sliding surface $\{\boldsymbol{\sigma}=\dot{\boldsymbol{\sigma}}=0\}$ (see [33] for further details).

Hence, the system trajectories evolve on $\mathcal{S}^{2}$ after a finite time and remains there in spite of the uncertainties. Using the control input with the proposed sliding vector, the Heisenberg system can be controlled in the sliding mode from the initial time instant since $\dot{\boldsymbol{\sigma}}(0)=\boldsymbol{\sigma}(0)=0$. Thus, the discontinuous control term maintains the system on the sliding surface by rejecting the effect of the uncertainties for $t \geq 0$ and avoids control singularities (i.e. $\|\mathbf{X}\| \neq 0$ ). Due to lemma 3.3, one obtains the practical stabilization of the state.

REMARK 6 It should be stressed that the $2^{\text {nd }}$ order sliding mode controller is not used here to remove the chattering phenomenon but to obtain the vanishing of a sliding vector that do not require the knowledge of $\dot{z}$ and $\dot{\mathbf{X}}$. Indeed, because of the new choice of the sliding vector, the discontinuous control is still applied on the variable $\mathbf{U}$, as in the first order sliding mode case. Actually, in both cases, integrators in the path of the usual control inputs of the Heisenberg system were added to alleviate the chattering phenomenon.

\section{Practical tracking problem for the extended Heisenberg system}

This control methodology can be proved also effective to solve a tracking problem. The interesting fact here is that one can track smooth $n+1$ dimensional trajectories by using only $n$-dimensional control input $\mathbf{U} \in \mathbb{R}^{n}$.

Let us consider a bounded and smooth reference trajectory $\boldsymbol{\Sigma}_{r}=\left[z_{r}, \mathbf{X}_{r}^{T}\right]^{T}$ which may not satisfy the system equation:

where $\mathbf{Y}_{r}=\dot{\mathbf{X}}_{r}-J_{2} \mathbf{X}_{r}$.

$$
\dot{z}_{r}=\mathbf{Y}_{r}^{T} J_{1} \mathbf{X}_{r}+\delta_{1}\left(\boldsymbol{\Sigma}_{r}, t\right)
$$

In this case, it is assumed that the skew symmetric matrices $J_{1}, J_{2}$ do not depend on the state, but with a few modifications our method can be applied to the general case, as well.

Let us define the modified tracking error as:

$$
\bar{\Xi}=\left[\bar{z}, \overline{\mathbf{X}}^{T}, \overline{\mathbf{Y}}^{T}\right]^{T}
$$

where $\overline{\mathbf{X}}=\mathbf{X}-\mathbf{X}_{r}, \overline{\mathbf{Y}}=\mathbf{Y}-\mathbf{Y}_{r}$ and where $\bar{z}$ is defined by:

$$
\bar{z}=z-z_{r}+\mathbf{X}_{r}^{T} J_{1} \mathbf{X}
$$

Differentiating the modified tracking error (41), one obtains:

$$
\begin{aligned}
\frac{d}{d t} \bar{z} & =\overline{\mathbf{Y}}^{T} J_{1} \overline{\mathbf{X}}+\Lambda_{1}(\boldsymbol{\Sigma}) \\
\frac{d}{d t} \overline{\mathbf{X}} & =\overline{\mathbf{Y}}+J_{2} \overline{\mathbf{X}}+\Lambda_{2}(\boldsymbol{\Sigma}) \\
\frac{d}{d t} \overline{\mathbf{Y}} & =\overline{\mathbf{U}}+\delta_{3}(\boldsymbol{\Xi})
\end{aligned}
$$

where

$$
\begin{aligned}
& \Lambda_{1}(\boldsymbol{\Sigma})=\delta_{1}(\boldsymbol{\Sigma})+F_{\bar{z}}(\boldsymbol{\Sigma}) \\
& \Lambda_{2}(\boldsymbol{\Sigma})=\delta_{2}(\boldsymbol{\Sigma}) \\
& F_{\bar{z}}(\boldsymbol{\Sigma})=\mathbf{X}_{r}^{T} J_{2} J_{1} \overline{\mathbf{X}}+\dot{\mathbf{X}}_{r}^{T} J_{1}\left(2 \overline{\mathbf{X}}+\mathbf{X}_{r}\right)+\mathbf{X}_{r}^{T} J_{1} J_{2} \mathbf{X}-\dot{z}_{r}+\mathbf{X}_{r}^{T} J_{1} \delta_{2}(\boldsymbol{\Sigma})
\end{aligned}
$$


The new control variable is $\overline{\mathbf{U}}=\mathbf{U}-\dot{\mathbf{Y}}_{r}$.

THEOREM 4.1 Let us consider an arbitrarily bounded and smooth reference trajectory $\left[z_{r}, \mathbf{X}_{r}^{T}\right]$. Assume that there exist a constant $1 \gg \epsilon>0$ such that $\|\overline{\mathbf{X}}(0)\|^{2}>\epsilon$, positive constants $k_{i}(i=$ $1, \ldots, 4)$ and a twice differentiable positive definite function $\varphi(\bar{z})$ such that $\frac{\|\overline{\mathbf{X}}(0)\|^{2}-\epsilon}{2} \geq \varphi(\bar{z}(0))$. Apply the integral second order sliding mode controller:

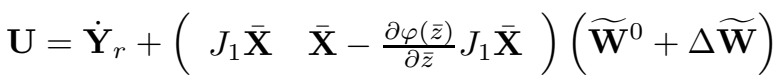

where

$$
\widetilde{\mathbf{W}}^{0}=-N(\overline{\boldsymbol{\Xi}})^{-1} \Gamma(\overline{\boldsymbol{\Xi}})
$$

and

with, for $j=1,2$

$$
\Delta \widetilde{\mathbf{W}}=\left[\Delta \widetilde{W}_{1}, \Delta \widetilde{W}_{2}\right]^{T}
$$

$$
\Delta \widetilde{W}_{j}=\left\{\begin{array}{l}
-N(\boldsymbol{\Xi})^{-1} \lambda_{m} \operatorname{sign}\left(\sigma_{j}\right), \text { if } \sigma_{j} \Delta_{\sigma_{j}} \leq 0 \\
-N(\boldsymbol{\Xi})^{-1} \lambda_{M} \operatorname{sign}\left(\sigma_{j}\right), \text { if } \sigma_{j} \Delta_{\sigma_{j}}>0
\end{array}\right.
$$

where the sliding vector $\boldsymbol{\sigma}$ is defined by equations (31)-(32) replacing $\mathbf{X}$ by $\overline{\mathbf{X}}$ and $z$ by $\bar{z}$. The gains are derived from condition (39) taking into account the additional disturbance $F_{\bar{z}}(\boldsymbol{\Xi})$. Then, there exist sufficiently large $\lambda_{m}$ and $\lambda_{M}$ such that the modified tracking error $[\bar{z}, \overline{\mathbf{X}}]^{T}$ is practically stable.

Proof. Since system (43)-(45) is similar to system (10)-(12), with the addition of disturbance $F_{\bar{z}}(\boldsymbol{\Sigma})$, which preserves the assumptions applied to the disturbance in system (10)-(12), one can apply Theorem 3.4. Hence, the practical stabilization of $\left[\bar{z}, \overline{\mathbf{X}}^{T}\right]^{T}$ is ensured in spite of the uncertainties. Furthermore, as $\mathbf{X}_{r}^{T} J_{1} \mathbf{X}_{r}=0$, the variable $\bar{z}$ can be expressed as:

$$
\bar{z}=z-z_{r}+\mathbf{X}_{r}^{T} J_{1} \overline{\mathbf{X}}
$$

Thus, the practical stability of $\left[\bar{z}, \overline{\mathbf{X}}^{T}\right]^{T}$ implies the practical stability of the tracking errors $\left(z-z_{r}\right)$ and $\left(\mathbf{X}-\mathbf{X}_{r}\right)$.

\section{Simulation results}

In this section, simulations of the practical stabilization and tracking of the extended Heisenberg system (10)-(12), using the designed control algorithms, are given for the case $n=2, J_{1}=\left[\begin{array}{cc}0 & -1 \\ 1 & 0\end{array}\right]$ and $J_{2}=\left[\begin{array}{ll}0 & 0 \\ 0 & 0\end{array}\right]$. This example is closely related to many mechanical systems like wheeled mobile robots. Nevertheless, one can easily obtain similar results for higher dimensional systems (i.e. $n>2$ ) by replacing $\mathbf{X}$ and $\mathbf{Y}$ by vectors of dimension $n$. In the following simulations, the sampling step and the integration steps are arbitrarily chosen to $\tau=10^{-3} \mathrm{~s}$. The parameter $\epsilon$, which is related to the desired accuracy of $\|\mathbf{X}\|^{2}$, is set to $\epsilon=10^{-4}$. Hence, the control objective is to stabilize $z$ to zero and $\|\mathbf{X}\|$ to $\sqrt{\epsilon}=10^{-2}$.

\subsection{Practical stabilization}

Here, the initial conditions are $z(0)=0.5, x_{1}(0)=1$ and $x_{2}(0)=-0.5$. It implies that $\|\mathbf{X}(0)\|^{2}=$ 1.25. Thus, Assumption 1 is satisfied. Furthermore, one can select the positive definite function $\varphi(z)=\frac{1}{2} z^{2}$ since $\frac{\|\mathbf{X}(0)\|^{2}-\epsilon}{2}=0.625 \geq \varphi(z(0))=0.125$. For comparison purpose, the parameters $k_{1}=k_{3}=2$ and $k_{2}=k_{4}=1$ which define the dynamics in sliding mode (i.e. see equation (21)) are chosen both for the sliding vector (16) and (31). 


\subsubsection{Without disturbance $\left(\delta_{1}=\delta_{2}=\delta_{3}=0\right)$}

Since Assumption 1 is satisfied and $\delta_{1}=\delta_{2}=0$, Theorem 3.2 can be applied in order to design a first order sliding mode controller which guarantees the practical stabilization of the system (10)(12). The control gain $K$ is tuned to achieve the finite time stabilization of the sliding vector (16) and is set to $K=5$.

The simulation results are depicted in Fig. 2. One can see that the sliding mode occurs from the initial time instant since $s_{1}$ and $s_{2}$ are in the range of $\tau=10^{-3}$ from $t=0$. Therefore, $z$ and $\psi$ fulfill the differential equations (21). Thus, Fig. 2 shows the exponential stability of $z$ and $\psi$. Furthermore, the time evolution of $z$ and $\psi$ are similar as $k_{1}=k_{3}=2$ and $k_{2}=k_{4}=1$. Since $z$ converges toward zero, the positive definite function $\varphi(z)$ vanishes. From equation (18), one can conclude that $\|\mathbf{X}\|$ exponentially converges to $\epsilon=10^{-2}$ (see Fig. 2).
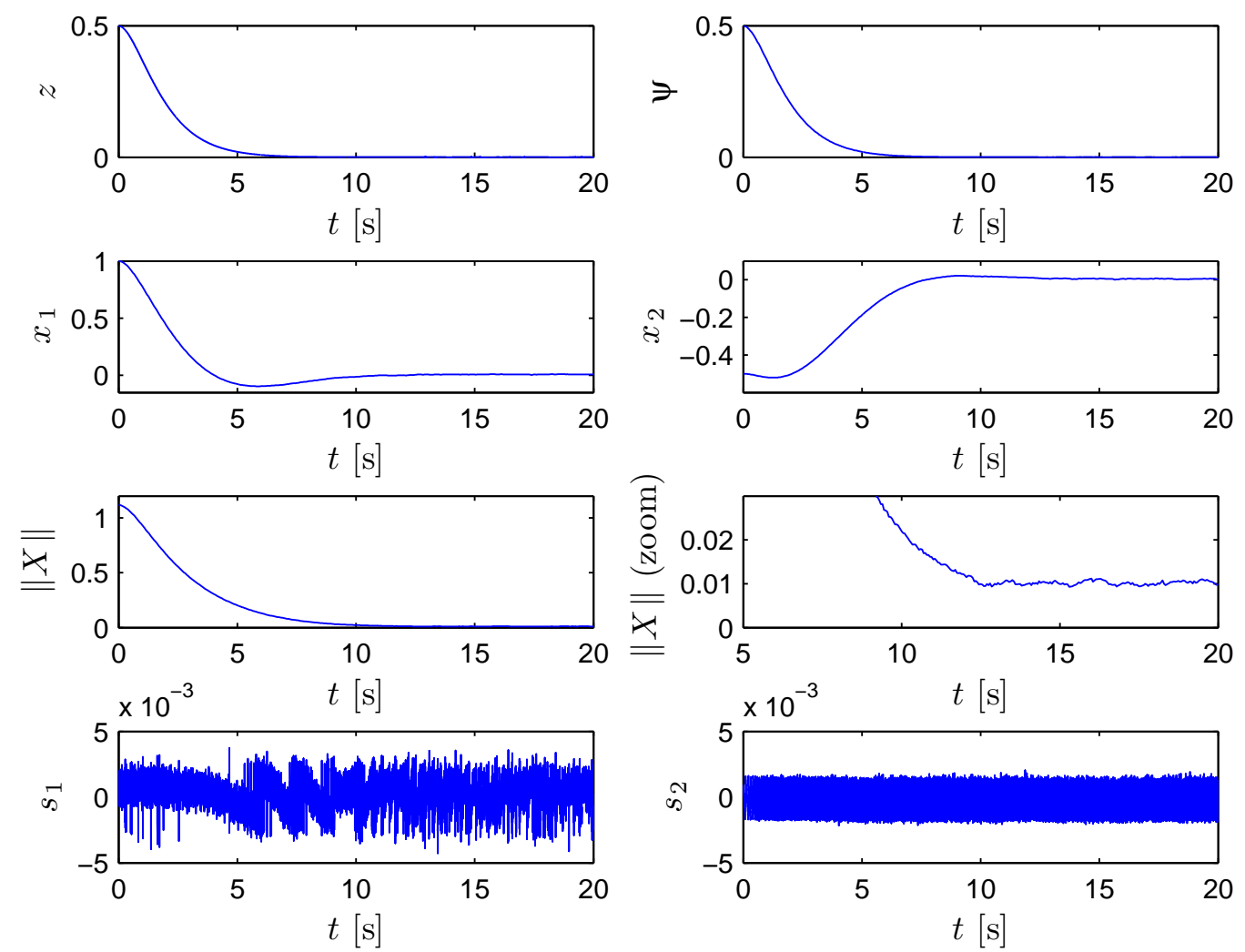

Figure 2: Practical stabilization of the unperturbed extended Heisenberg system using first order integral sliding mode control

\subsubsection{With matched and unmatched disturbances}

Here, some perturbations have been added:

$$
\delta_{1}=\delta_{2}=\delta_{3}=0.1 \sin (2 \pi t)
$$


Note that since these perturbations do not verify the matching condition one cannot apply Theorem 3.2. Indeed, using a first order sliding mode controller, the desired accuracy of $\|\mathbf{X}\|^{2}$, i.e. $\epsilon$, cannot be arbitrary chosen and depends on the size of the unmatched uncertainties. In this case, $\epsilon$ must be increased to $\epsilon=2 \cdot 10^{-3}$ to avoid singularities in the control. Hence, the steady state errors of $x_{1}$ and $x_{2}$ become large.

Nevertheless, Theorem 3.4 can be applied in order to design a second order sliding mode controller which guarantees the practical stabilization of the system (10)-(12). The control gains $\lambda_{m}$ and $\lambda_{M}$ are tuned to achieve the finite time stabilization of the sliding vector (31) and are set to $\lambda_{m}=5$ and $\lambda_{M}=20$.

The simulation results are depicted in Fig. 3. One can see that the sliding mode occurs from the initial time instant since $\sigma_{1}$ and $\sigma_{2}$ are in the range of $\tau^{2}=10^{-6}$ from $t=0$. Therefore, $z$ and $\psi$ fulfill the differential equations (21). Thus, the exponential stability of $z$ and $\psi$ is ensured in spite of the unmatched uncertainties. From equation (18), one can conclude that $\|\mathbf{X}\|$ exponentially converges to $\epsilon=10^{-2}$ (see Fig. 3). As expected, the second order sliding mode controller efficiently corrects the undesirable deviations due to the uncertainties.
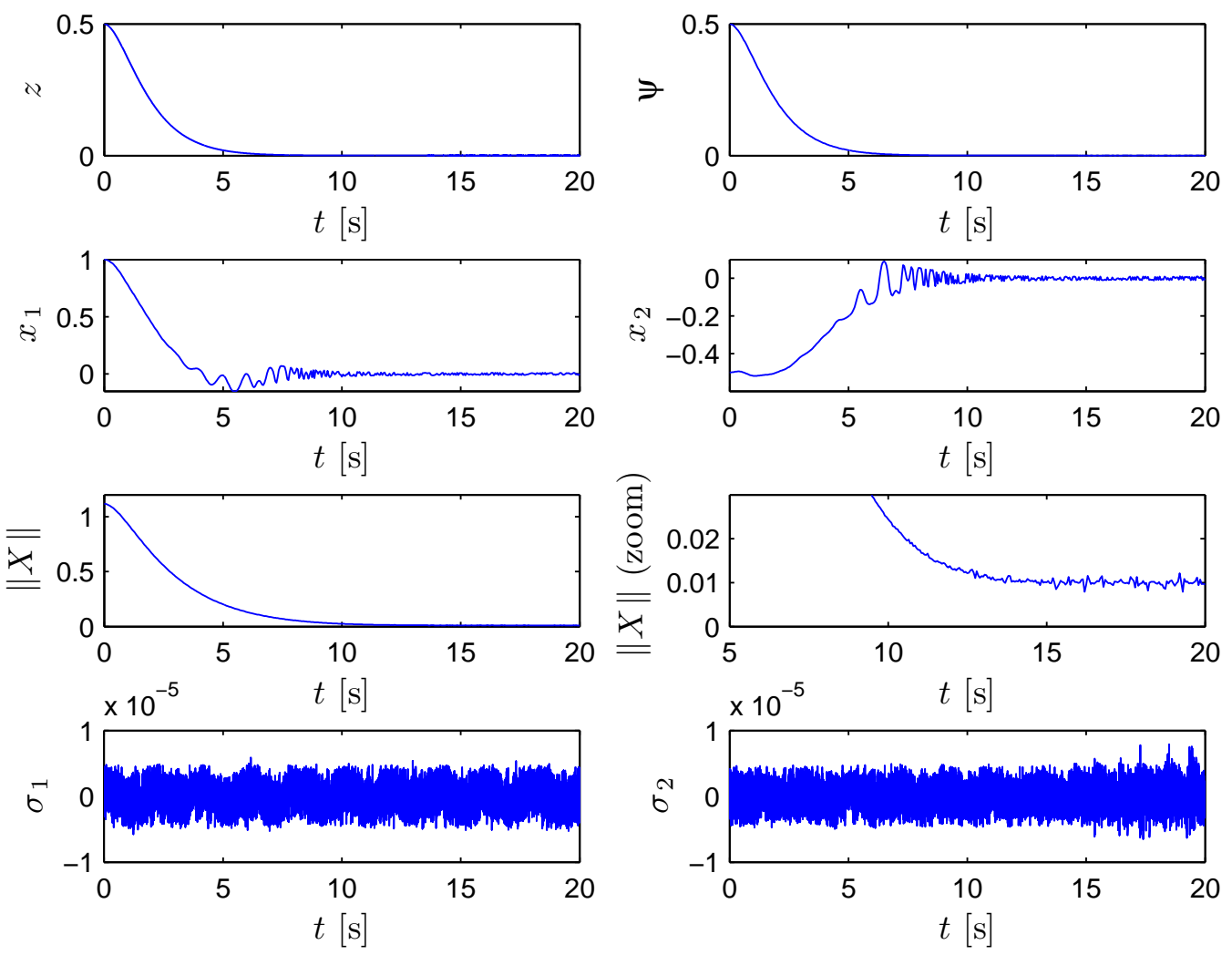

Figure 3: Practical stabilization of the perturbed extended Heisenberg system using second order integral sliding mode control 


\subsection{Practical tracking with matched and unmatched disturbances}

The control objective is to robustly steer the extended Heisenberg system (10)-(12) from a given initial position to a neighborhood of the trajectory:

$$
\begin{aligned}
z_{r} & =0.01 t \\
\mathbf{X}_{r} & =\left[\begin{array}{l}
0.2 \cos (t) \\
0.2 \sin (t)
\end{array}\right]
\end{aligned}
$$

One can note that this trajectory does not satisfy equation (40). Indeed, $\dot{z}_{r}=0.01 \neq \mathbf{Y}_{r}^{T} J_{1} \mathbf{X}_{r}=$ 0.04. This example is related to the case that the motion planner optimally interpolates a set of stored desired waypoints that the system must follow, without taking into account the nonholonomic constraint (see [35] for instance). It may be useful in order to reduce the computing time for the generation of the reference trajectory.

The initial conditions $z(0)=0.5, x_{1}(0)=1$ and $x_{2}(0)=-0.5$ are taken. It implies that $\bar{z}(0)=z(0)-z_{r}(0)+\mathbf{X}_{r}^{T}(0) J_{1} \mathbf{X}(0)=0.6$ and $\|\overline{\mathbf{X}}(0)\|^{2}=0.89$. Thus, Assumption 1 is satisfied. Furthermore, one can select the positive definite function $\varphi(\bar{z})=\frac{1}{2} \bar{z}^{2}$ since $\frac{\|\overline{\mathbf{X}}(0)\|^{2}-\epsilon}{2}=0.445 \geq$ $\varphi(\bar{z}(0))=0.18$. The parameters $k_{1}=k_{2}=k_{3}=k_{4}=4$ are chosen for the sliding vector (31). Theorem 4.1 can be applied in order to design a second order sliding mode controller which guarantees the practical stabilization of the system (43)-(45). The control gains $\lambda_{m}$ and $\lambda_{M}$ are tuned to achieve the finite time stabilization of the sliding vector (31) and are set to $\lambda_{m}=5$ and $\lambda_{M}=20$.

The simulation results are depicted in Fig. 4. One can see that the sliding mode occurs from the initial time instant since $\sigma_{1}$ and $\sigma_{2}$ are in the range of $\tau^{2}=10^{-6}$ from $t=0$. Thus, the exponential stability of $\bar{z}$ and $\psi$ is ensured in spite of the unmatched uncertainties. Since $\bar{z}$ converges toward zero, the positive definite function $\varphi(\bar{z})$ vanishes. One can conclude that $\|\overline{\mathbf{X}}\|$ exponentially converges to $\epsilon=10^{-2}$. Hence, it implies the practical stability of the tracking errors $\left(z-z_{r}\right)$ and $\left(\mathbf{X}-\mathbf{X}_{r}\right)$ (see Fig. 4$)$.

\section{$6 \quad$ Experimental results}

In this section, experimental results of the stabilization and the tracking of a wheeled mobile robot using the proposed strategies are given ${ }^{2}$. Note that due to the presence of uncertainties, one can only apply the second order sliding mode controller since the use of the first order sliding mode controller does not enable to take into account the unmatched uncertainties and would lead to poor performances. In addition to the good robustness properties, the second order sliding mode controller has the important feature of being applicable to both the practical stabilization and tracking problems.

\subsection{Experimental configuration}

The developed controller is implemented on an experimental mobile robot called Pekee, shown in Fig. 5, which is known to be subject to input time-delay, measurement time-delay, measurement noise and parameter variations. Pekee is an open robotic platform designed by Wany Robotics (Montpellier, France) and is driven by two independent motorized wheels, with a free rotating caster wheel at the back. This layout offers substantial mobility. The positions of the two independently controlled driving motors are measured via two encoders (180 impulses/wheel-turn). Based on the motor positions, the linear and angular velocities are calculated via a filtered backward difference algorithm. A numerical integration routine enables to obtain the actual values of the coordinates $\mathbf{q}$ of the mobile robot. An Intel 486 micro-processor running at $75 \mathrm{MHz}$ operating under linux

\footnotetext{
${ }^{2}$ The corresponding experimental results are available on: http://www.isen.fr/ sst_lille/videos.htm.
} 

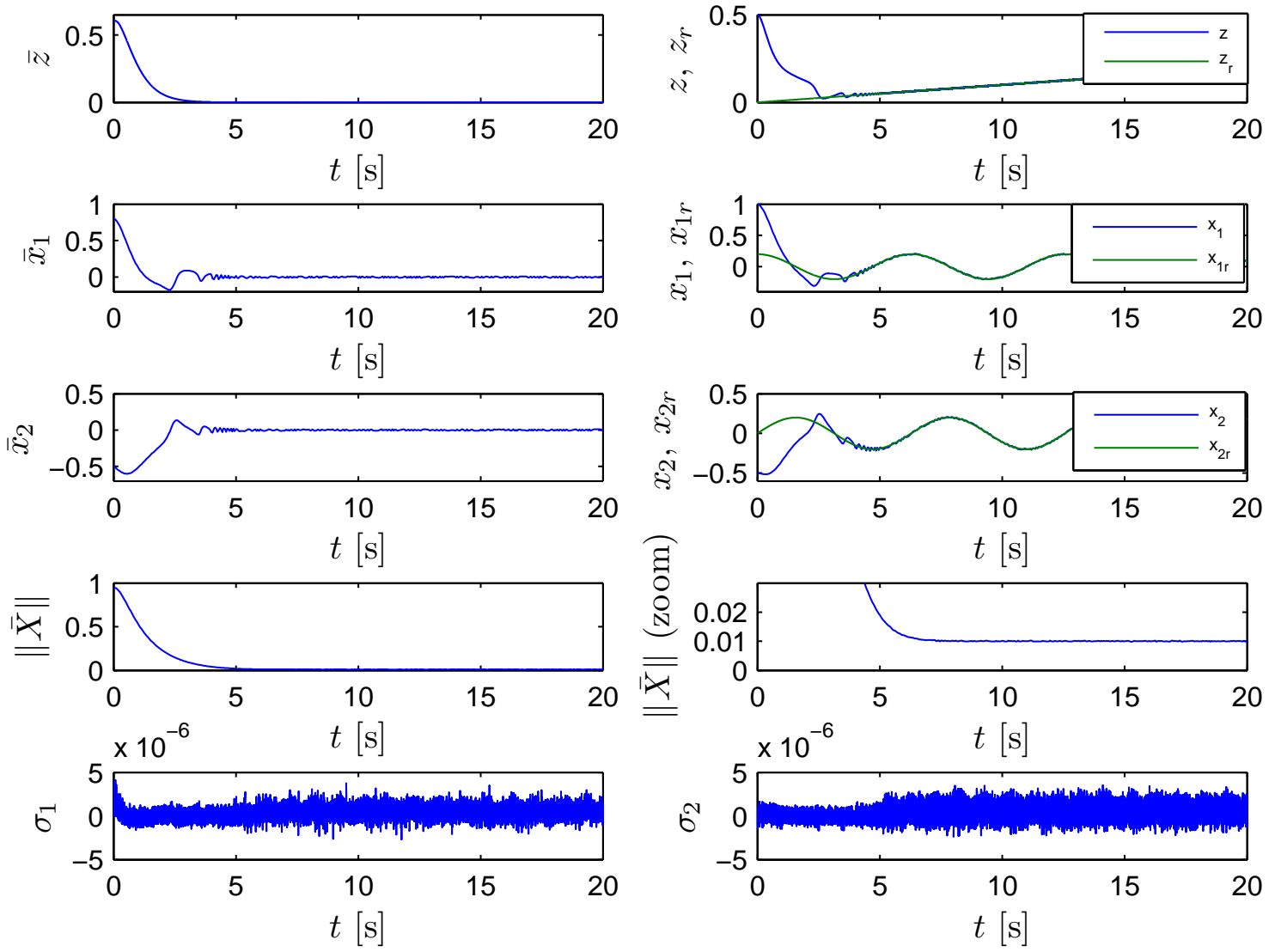

Figure 4: Tracking of the perturbed Heisenberg system using second order integral sliding mode control

real time hosts the second order integral sliding mode controller written in $\mathrm{C}$ language. Since the localization of the robot is made though the embedded computer which has poor performances, the processing rate of the data acquisition limits the sampling time for the controller. It is set to $\tau=0.1 \mathrm{~s}$, which is identical to the processing rate. The radius of the wheels is $0.0072 \mathrm{~m}$ and the length of the axis between the wheels is $0.225 \mathrm{~m}$. The maximum linear speed and angular velocities are $1.5 \mathrm{~m} / \mathrm{s}$ and $1.5 \mathrm{rad} / \mathrm{s}$, respectively.

The parameter $\epsilon$, which is related to the desired accuracy of $\|\mathbf{X}\|^{2}$ (that is to say $x, y$ and $\theta$ through relation $(5))$, is set to $\epsilon=0.02$.

\subsection{Practical stabilization}

At the initial time $t=0 \mathrm{~s}$, the position of the mobile robot is $x(0)=1 \mathrm{~m}, y(0)=1 \mathrm{~m}$ and $\theta(0)=3 \mathrm{deg}$. The global invertible transformation (5) yields $z(0) \simeq 1.9, x_{1}(0)=0.1$ and $x_{2}(0) \simeq 1.1$, i.e. $\|\mathbf{X}(0)\|^{2} \simeq 1.2$ and Assumption 1 is satisfied. Furthermore, one can select the positive definite function $\varphi(z)=\frac{1}{10} z^{2}$ since $\frac{\|\mathbf{X}(0)\|^{2}-\epsilon}{2} \simeq 0.59 \geq \varphi(z(0)) \simeq 0.36$. Then, Theorem 3.4 is applied in order to design a second order sliding mode controller which guarantees the practical stabilization 


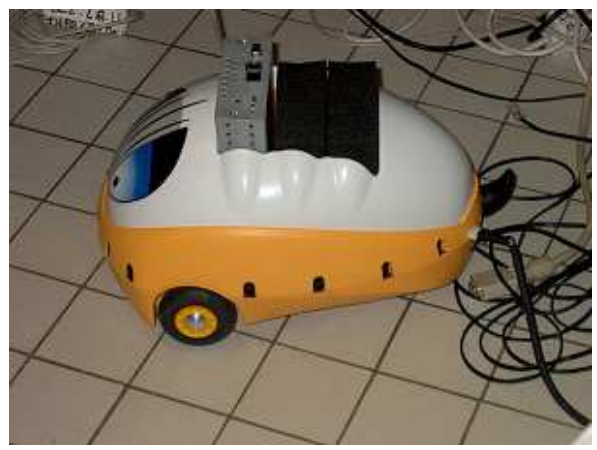

Figure 5: Pekee Mobile Robot

of system (9), i.e. the exponential stability of $z$ to zero and of $\|\mathbf{X}\|$ to $\sqrt{\epsilon} \simeq 0.14$. It implies the practical stability of the state $\mathbf{q}$ due to relation (5). The control gains are tuned in order to achieve good results and to respect the limitations on the linear and angular velocities. The parameters that resulted in good performances are:

$$
k_{1}=1, \quad k_{2}=1, \quad k_{3}=2, \quad k_{4}=1, \quad \lambda_{m}=1 \quad \text { and } \lambda_{M}=5
$$

The position/orientation of the mobile robot and the associated linear and angular velocities are shown in Fig. 6(a) and 6(b), respectively. Based on Fig. 6(a), it is clear that practical stability of the state is obtained in a neighbourhood of the origin defined by the choice of $\epsilon$ (note that in practice, the lower value of $\epsilon$ is limited by the sensor accuracy). As it can be seen on Fig. 6(b), the chattering phenomenon is removed since the discontinuities act on the time derivative of the velocities. The actual trajectory of the mobile robot is depicted in Fig. 6(c) and shows the parking manoeuvres.

\subsection{Practical tracking}

The control objective is to robustly steer the nonholonomic mobile robot from a given initial position to a neighborhood of the reference time-varying trajectory given by the following reference linear and angular velocities:

$$
\left\{\begin{array}{l}
w_{l, r}=0.3(1-\exp (-t)) \\
w_{a, r}=0.1 \sin (0.5 t)
\end{array}\right.
$$

The resulting reference time-varying Cartesian position and orientation are depicted in Fig. 7(c). The linear velocity is related to a first order dynamic system with appropriate parameters in order to take into account the robot specifications. The angular velocity is chosen as a sinusoidal function. Hence, this problem is not trivial.

REMARK 7 It should be noted that since the system (4) is flat (see [36]), a collision-free optimal reference trajectory can be generated on-line (see for instance [37]).

At the initial time $t=0 \mathrm{~s}$, the position of the mobile robot is $x(0)=0.05 \mathrm{~m}, y(0)=0 \mathrm{~m}$ and $\theta(0)=4 \mathrm{deg}$. The global invertible transformation (5) yields $z(0) \simeq-0.008, x_{1}(0) \simeq 0.16$ and $x_{2}(0) \simeq 0.05$, i.e. $\|\mathbf{X}(0)\|^{2} \simeq 0.028$. Thus, Assumption 1 is satisfied. Furthermore, one can select the positive definite function $\varphi(z)=\frac{1}{2} z^{2}$ since $\frac{\|\mathbf{X}(0)\|^{2}-\epsilon}{2} \simeq 0.004 \geq \varphi(z(0)) \simeq 3.10^{-5}$. Then, Theorem 4.1 is applied in order to design a second order sliding mode controller which guarantees the practical stabilization of $\left[\bar{z}, \overline{\mathbf{X}}^{T}\right]^{T}$ and of the tracking errors of the original state variables due 


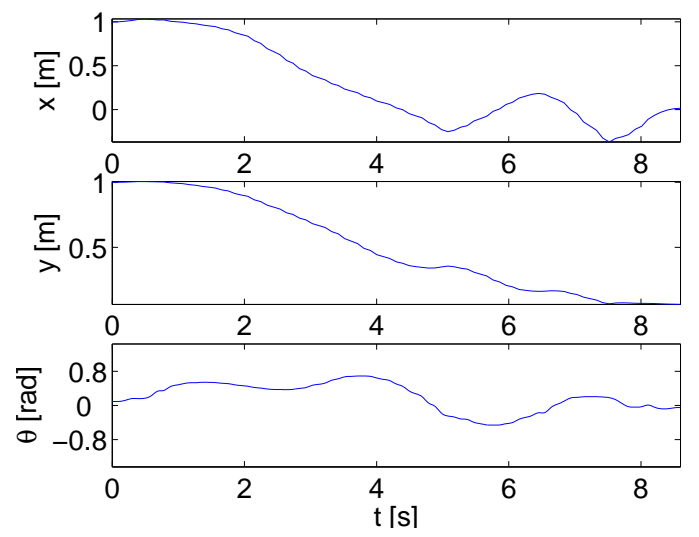

(a)
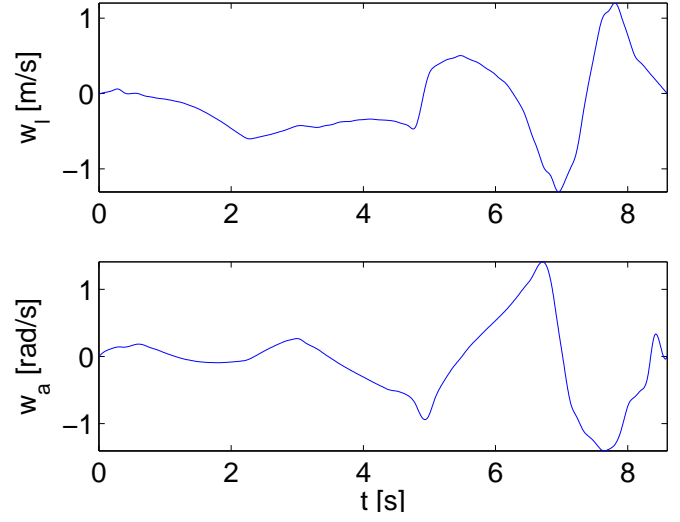

(b)

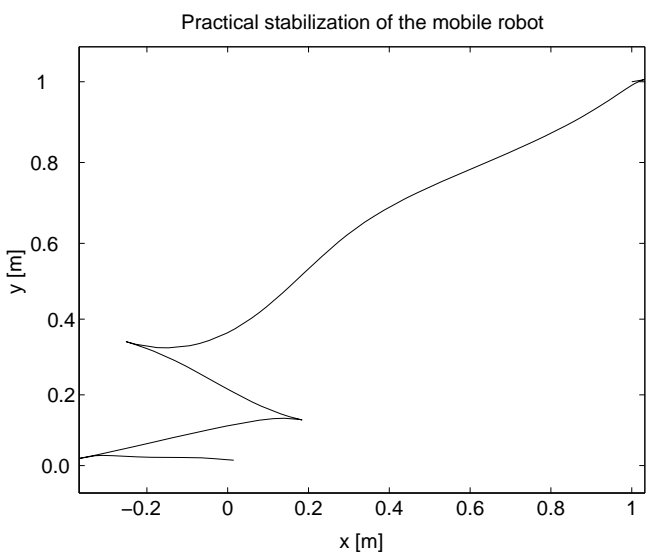

(c)

Figure 6: Practical stabilization of the mobile robot

to relation (5). In order to highlight the adaptability of the controller, the control parameters are not retuned (i.e. parameters given in (50)).

The position/orientation tracking errors of the mobile robot and the associated linear and angular velocities are shown in Fig. 7(a) and 7(b), respectively. It can be seen in Fig. 7(a) that practical stabilization around the reference trajectories is obtained, even if the latter does not fulfilled the dynamics of the mobile robot. The chattering phenomenon is also removed (see Fig. 7(b)). The actual trajectory and the reference trajectory of the mobile robot are depicted in Fig. 7(c). As expected, the practical stabilization of the tracking errors of the mobile robot is guaranteed.

\section{Conclusion}

A robust sliding mode control algorithm using an integral sliding variable has been introduced in this paper and applied to solve the practical stabilization and tracking problems for a class of a perturbed extended Heisenberg system with additional integrators in the control input path. The proposed controller has particularly the following advantages: 1) good robustness properties against unmatched uncertainties can be obtained 2) the chattering phenomenon is removed 3) 


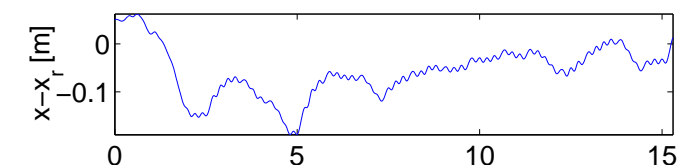

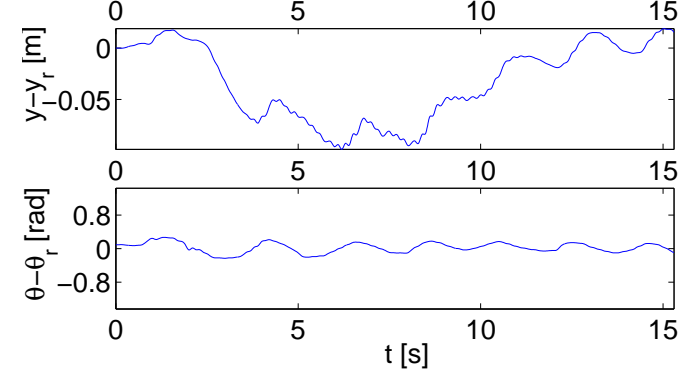

(a)
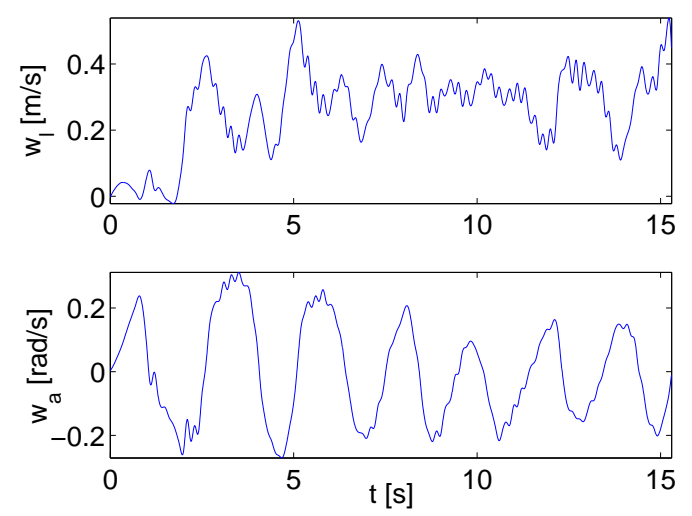

(b)

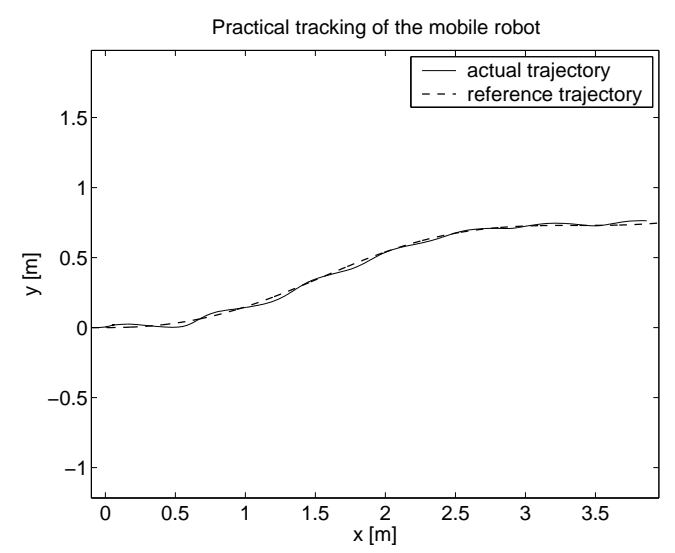

(c)

Figure 7: Practical tracking of the mobile robot

practical stabilization of the tracking errors can be obtained without adding restrictive conditions on the reference trajectory. Furthermore, an interesting and original application of integral sliding mode has been highlighted: it is possible to avoid some control singularities that could appear during the reaching phase with classical sliding mode control laws. To demonstrate the efficiency of the proposed controller, experimental results on a wheeled mobile robot have been given for solving both the parking and trajectory tracking problems.

\section{References}

[1] Kolmanovsky, I., and McClamroch, N. H., 'Developments in nonholonomic control problems', IEEE Control Syst. Mag., 1995, 15, pp. 20-36.

[2] Brockett, R. W. , 'Asymptotic stability and feedback stabilization', in Differential Geometric Control Theory, R. Brockett, R. Millman and H. Sussmann, eds., Boston: Birkhauser, 1983, pp. 181-191.

[3] Pomet, J. B., 'Explicit design of time-varying stabilizing control laws for a class of controllable systems without drift', Systems and Control Letters, 1992, 18, pp. 147-158. 
[4] Murray, R., and Sastry, S., 'Nonholonomic Motion Planning: Steering Using Sinusoids', IEEE Trans. on Automat. Control, 1993, 38, pp. 700-716.

[5] Jiang, Z. P., and Nijmeijer, H., 'Tracking control of mobile robots: a case study in backsteeping', Automatica, 1997, 33, pp. 1393-1399.

[6] Astolfi, A., 'Discontinuous control of nonholonomic systems', Systems and Control Letters, 1996, 27, (1), pp. 37-45.

[7] Bloch, A. M., and Drakunov, S. V., 'Stabilization and tracking in the nonholonomic integrator via sliding modes', System and Control Letters, 1996, 29, (2), pp. 91-99.

[8] Bloch, A. M., Drakunov, S. V., and Kinyon, M. K., 'Stabilization of Nonholonomic Systems Using Isospectral Flows', SIAM J. on Control and Optimization, 2000, 38, (3), pp. 855-874.

[9] Hespanha, J. P., Liberzon, D., and Morse, A. S., 'Logic-based switching control of a nonholonomic system with parametric modeling uncertainty', Systems and Control Letters, 1999, 38, pp. $167-177$.

[10] Khennouf, H., and Canudas de Wit, C., 'On the construction of stabilizing discontinuous controllers for nonholonomic systems', Proc. IFAC Nonlinear Control Systems Design Symposium, Tahoe City, CA, 1995, pp. 747-752.

[11] Marchand, N., and Alamir, M., 'Discontinuous exponential stabilization of chained form systems', Automatica, 2003, 39, pp. 343-348.

[12] Prieur, C., and Astolfi, A., 'Robust stabilization of chained systems via hybrid control', IEEE Trans. Automat. Control, 2003, 48, pp. 1768-1772.

[13] Floquet, T., Barbot, J. P., and Perruquetti, W., 'Higher-order sliding mode stabilization for a class of nonholonomic perturbed systems', Automatica, 2003, 39, (6), pp. 1077-1083.

[14] Prieur, C., and Trelat, E., 'Robust optimal stabilization of the Brockett integrator via a hybrid feedback', Math. Control Signals Systems, 2005, 17, pp. 201-216.

[15] Valtolina, E., and Astolfi, A., 'Local robust regulation of chained systems', Systems Control Letters, 2003, 49, (3), pp. 231-238.

[16] Utkin, V. I., 'Variable structure systems with sliding modes', IEEE Trans. on Automat. Control, 1977, 22, (2), pp. 212-222.

[17] Edwards, C., and Spurgeon, S. K., 'Sliding Mode Control: Theory and Applications', in Systems and Control Book Series, Taylor and Francis, 1998.

[18] Perruquetti, W., and Barbot, J. P., 'Sliding mode control in engineering', Control Eng. Series, Marcel Dekker, 2002.

[19] Emel'yanov, S. V., Korovin, S. K., and Levantovsky, L. V., 'Higher Order Sliding Modes in the Binary Control System', Soviet Physics, 1986, 31, (4), pp. 291-293.

[20] Drakunov, S. V., and Utkin, V., 'Sliding Mode Control in Dynamic Systems', Int. J. of Control, 1992, 55, (4), pp. 1029-1037.

[21] Drakunov, S. V., Floquet, T., and Perruquetti, W., 'Stabilization and tracking control for an extended Heisenberg system with a drift', Systems and control Letters, 2005, 54, pp. 435-445.

[22] Utkin, V. I., and Shi, J.,'Integral sliding mode in systems operating under uncertainly conditions', Proc. of the $35^{\text {th }}$ Conf. on Decision and Control, 1996, pp. 4591-4596. 
[23] Cao, W. J., and Xu, J. X., 'Nonlinear integral-type sliding surface for both matched and unmatched uncertain systems', IEEE Trans. Automatic Control, 2004, 49, (8), pp. 1355-1360.

[24] Laghrouche, S., Plestan, F., and Glumineau, A., 'Higher order sliding mode control based on integral sliding surface', Automatica, 2007, 43, (3), pp. 531-537.

[25] Castaños, F., and Fridman, L., 'Analysis and design of integral sliding manifolds for systems with unmatched uncertainties', IEEE Trans. Automatic Control, 2006, 51, (5), pp. 853-858.

[26] Escobar, G., Ortega, R., and Reyhanoglu, M., 'Regulation and tracking of the nonholonomic double integrator: a Field-oriented control approach', Automatica, 1998, 34, (1), pp. 125-131.

[27] Leonhard, W., 'Control of electrical drives', Springer-Verlag, Berlin, 1985.

[28] Dixon, W. E., Jiang, Z. P., and Dawson, D. M., 'Global exponential setpoint control of wheeled mobile robots: a Lyapunov approach', Automatica, 2000, 36, (11), pp. 1741-1746.

[29] Brockett, R. W. , 'Control theory and singular Riemannian geometry', in New Directions in Applied Mathematics, P. Hilton and G. Young, eds., New York: Springer-Verlag, 1981, pp. $11-27$.

[30] Canudas de Wit, C., and Sordalen, O., 'Exponential stabilization of mobile robots with nonholonomic constraints', IEEE Trans. on Automatic Control, 1992, 37, (11), pp. 1791-1797.

[31] Isidori, A., 'Nonlinear control systems (2nd ed.)', New York: Springer, 1989.

[32] Bartolini, G., Ferrara, A., Levant, A., and Usai, E., 'On second order sliding mode controllers', in Variable structure systems, sliding mode and nonlinear control, Lecture Notes in Control and Information Sciences, Springer, 1999.

[33] Fridman, L., and Levant, A., 'Higher-order sliding mode', in Sliding Mode Control in Engineering, W. Perruquetti and J. P. Barbot (Editors), Marcel Dekker, 2002, pp. 53-101.

[34] Levant, A., 'Higher order sliding modes, differentiation and output feedback control', Int. J. of Control, 2003, 76, pp. 924-941.

[35] Chen, Y. and Huang, J. 'A new computational approach to solving a class of optimal control problems', Int. J. of Control, 1993, 58, pp. 1361-1383.

[36] Fliess, M., Lévine, J., Martin, P., and Rouchon, P., 'Flatness and defect of nonlinear systems: introductory theory and examples', Int. J. of Control, 1995 61, (6), pp. 1327-1361.

[37] Defoort, M., Palos, J., Kökösy, A., Floquet, T., and Perruquetti, W, 'Performance-based reactive navigation for nonholonomic mobile robots', Robotica, In press. 